\title{
Predictive Processing and the Problem of (Massive) Modularity
}

\author{
Egil Asprem
}

Stockholm University

A theoretical shift is underway in the cognitive science of religion. As an increasing number of researchers are turning to the neurocognitive theory of predictive processing (e.g. Schjoedt et al. 2013; Nielbo \& Sørensen 2013; Andersen et al. 2014; Hermans 2015; Taves and Asprem 2017; Asprem 2017; Schjoedt \& Andersen 2017; van Elk \& Aleman 2017), aspects of the CSR "standard model" are coming under pressure. Andersen's target article is an excellent example of this trend. Its critique of agency detection illustrates what stands to be gained by making this theoretical shift, but it also brings to light a budding conflict with the discipline's foundations in evolutionary psychology. As Andersen demonstrates, the predictive processing account offers a coherent explanatory picture, a framework for generating and testing hypotheses experimentally, and appears to solve remaining inconsistencies in empirical work on HADD. It also points out a clear role for humanist scholars, by highlighting the place of "culture" and prior learning in perception. However, this focus on learning also opens up a discussion about the role of innate, functionally specialized modules selected for in humanity's ancestral environments. Can this apparent conflict be solved?

The success of conceptual shifts is judged as much by what is rejected as what is introduced. Conceptual shifts tend to involve "Kuhn loss" - when an older paradigm successfully explained some phenomenon which the new paradigm does not (Kuhn 1970, 99-100). Sometimes, new paradigms have to look backward and reconsider older explanations in order to make progress (see Midwinter \& Janssen 2012). While I am enthusiastic about the theoretical reorientation suggested by predictive processing, it is important not to throw out the baby with the bathwater. The bathwater may have become stale and murky by the expanding number of specialized modules, but in it swims a precious baby that must be saved: the idea that minds are created and shaped by natural selection. 
On first glance, two aspects of Andersen's account of agency detection challenge these foundations: (1) the role of domain-general over domain-specific processing, and (2) the role of top-down effects based on prior experience and learning. The first appears to conflict with functional specialization, while the second questions the innateness of modular processing.

Regarding the first, there is a crucial distinction to be made between the Bayesian algorithm, its implementation(s) in the brain, and the overall architecture of the cognitive system (cf. Clark 2013, 194). Consider old-fashioned Fodorian modularity (Fodor 1983): we might agree that the sensory modules at the cognitive periphery enjoy some degree of domain-specificity (in the sense of processing different kinds of stimuli), while holding that each modality processes those stimuli in a probabilistic, Bayesian way. Although the encapsulation of these sensory modalities has come into question due to cross-modal and cognitive penetrability effects - nicely accounted for by predictive processing (Clark 2016; Hohwy 2013) - there is clearly room for some degree of functional specialization, both in the neural implementation and the wider architecture of the predictive mind.

What then about innateness? The growing evidence of cognitive penetrability and top-down learning effects stressed by Andersen's account of agent detection clearly complicates the picture of robustly innate modules - and the CSR constructs based on them (e.g. HADD, $\mathrm{MCl}$ concepts). However, one should note that what massive modularity theorists propose are innate learning systems based on inbuilt biases (Tooby \& Cosmides 1992). Things may therefore not be as black and white as Andersen presents it when suggesting that we should ditch the very idea of HADD because the domain-general predictive algorithm does not provide a privileged place for agents (pp. [15, 17]). This all hinges on how prior probabilities are implemented in the cognitive system: If they result from experience alone (i.e. an organism's past attempts at predicting its environment), then the mind would, indeed, be an unbiased blank slate as far as agents are concerned. But nothing in the predictive processing story forces us to adopt this particular view. In fact, there are good reasons to think that some priors should be innate and evolved rather than learned by each individual organism. The so-called "dark room" problem exemplifies this: If cognition is all about minimizing surprises (prediction error), how come we don't tend to isolate ourselves in dark, quiet caves and let the brain simulate itself to near perfection? The 
short answer is that the brain does not expect a complete absence of sensory stimuli: that is why sensory deprivation may induce hallucinatory experiences (e.g. Corlett, Frith \& Fletcher 2009), an effect well-known to religious practices (see e.g. Ustinova 2009). So where do these basic expectations come from? From natural selection: if we didn't expect certain kinds of stimuli to begin with we would crawl up in dark caves and die (cf. Friston 2013, 213).

Andy Clark also opens the door for innate priors of various kinds: in the shape of "hyperpriors" ("priors upon priors", organizing our experience in a Kant-like manner; Clark 2016, 175) and "embodied biases" (evolved physiological structure functioning as inbuilt models of the world; ibid., 176). If we start thinking about priors not simply as what has been learned in previous iterations of predictive sensory-motor engagement with the world, but also as implemented by evolved physiology, it is (still) not inherently implausible that (say) an agent hypothesis for certain types of stimuli might come with a higher prior probability by natural design.

As long as such innate priors can be tweaked, shaped, strengthened or weakened by cultural variation, however, their existence make little difference to Andersen's methodological argument. But in the pursuit of a unified theoretical framework for CSR, we should seek to reconceptualize "modularity" in predictive processing terms rather than throw it out altogether. One possible approach is illustrated by van Elk and Aleman (2017), who seek a unified, predictive processing view of how several specific brain mechanisms associated with specific tasks (e.g. the "theory-of-mind network" and "mentalizing about gods") collectively account for phenomena deemed religious/spiritual. Approaches of this type hold great promise in connecting neurocognitive proximate mechanisms with evolutionary distal causes.

\section{References:}

Andersen, M., U. Schjoedt, K. L. Nielbo, \& J. Sørensen. (2014). Mystical experience in the lab. Method and Theory in the Study of Religion, 26, 217-245.

Asprem, E. (2017). Explaining the esoteric imagination: towards a theory of kataphatic practice. Aries, 17(1), 17-50. 
Open peer commentary on the target article: Marc Andersen, "Predictive Coding in Agency Detection". Religion, Brain \& Behavior (2017), doi: https://doi.org/10.1080/2153599X.2017.1387594

Clark, A. (2013). Whatever next? Predictive brains, situated agents, and the future of cognitive science. Behavioral and Brain Sciences, 36(3), 181-204.

Clark, A. (2016) Surfing uncertainty: Prediction, action, and the embodied mind. Oxford: Oxford University Press.

Corlett, P. R., Frith, C. D. \& Fletcher, P. C. (2009). From drugs to deprivation: A Bayesian framework for understanding models of psychosis. Psychopharmacology (Berlin), 206(4), 515-30.

Elk, M. van, and A. Aleman. (2017). Brain mechanisms in religion and spirituality: An integrative predictive processing framework. Neuroscience \& Biobehavioral Reviews, 73, 359-378.

Fodor, J. (1983). The modularity of mind. Cambridge: MIT Press.

Friston, K. (2013). Active inference and free energy. Behavioral and Brain Sciences, 36(3), 212-213.

Hermans, C. A. M. (2015). Towards a theory of spiritual and religious experiences: A building block approach of the unexpected possible. Archive for the Psychology of Religion, 37, 141-167.

Hohwy, J. (2013). The predictive mind. Oxford: Oxford University Press.

Kuhn, T. 1970. The structure of scientific revolutions. $2^{\text {nd }}$ ed. Chicago: University of Chicago Press.

Midwinter, C., \& Janssen, M. (2012). Kuhn losses regained: Van Vleck from spectra to susceptibilities. arXiv: 1205.0179v1 [physics.hist-ph].

Nielbo, Kristoffer L. \& Jesper Sørensen. (2013). Prediction error during functional and non-functional action sequences: A computational exploration of ritual and ritualized event processing. Journal of Cognition and Culture, 13 (3-4), 347-365.

Schjoedt, U., \& M. Andersen. (2017). How does religious experience work in predictive minds? Religion, Brain \& Behavior, doi: 0.1080/2153599X.2016.1249913.

Schjoedt, U., Sørensen, J., Nielbo, L. K., Xygalatas, D., Mitkidis, P., \& Bulbulia, J. (2013). The resource model and the principle of predictive coding: A framework for analyzing proximate effects of ritual. Religion, Brain \& Behavior, 3(1), 79-86.

Taves, A. \& E. Asprem (2017). Experience as event: Event cognition and the study of (religious) experience. Religion, Brain \& Behavior, 7(1), 43-62.

Tooby, J. \& Cosmides, L. (1992). The psychological foundations of culture. In J. Barkow, L. Cosmides \& J. Tooby (Eds.), The adapted mind: Evolutionary psychology and the generation of culture (pp. 19-135). New York: Oxford University Press.

Ustinova, Yulia (2009). Caves and the ancient Greek mind: Descending underground in the search for ultimate truth. Oxford: Oxford University Press. 